\title{
Coincidence of Some Classes of Universal Functions
}

\author{
Emmanuel S. KATSOPRINAKIS \\ University of Crete \\ Department of Mathematics \\ Knossou Ave. \\ GR-714 09 Heraklion, Crete - Greece \\ katsopr@math.uoc.gr
}

Received: February 25, 2008

Accepted: June 9, 2008

\begin{abstract}
Let $\Omega$ be a domain in the complex plane such that $\Omega$ satisfies appropriate geometrical and topological properties. We prove that if $f$ is a holomorphic function in $\Omega$, then its Taylor series, with center at any $\xi \in \Omega$, is universal with respect to overconvergence if and only if its Cesàro $(C, k)$-means are universal for any real $k>-1$. This is an extension of the same result, proved recently by F. Bayart, for any integer $k \geq 0$. As a consequence, several classes of universal functions introduced in the related literature are shown to coincide.

Key words: Universal series, overconvergence, Taylor series, Cesàro means, Ostrowski gaps.

2000 Mathematics Subject Classification: 30B30, 30B10, 30E10.
\end{abstract}

\section{Introduction}

The first example of a universal Taylor series in the complex plane $\mathbb{C}$ was defined by Seleznev in [32]. He proved that there exists a sequence of complex numbers $\left(a_{n}\right)$ such that, for any compact set $K \subset \mathbb{C}$ with $0 \notin K$ and connected complement and for any function $h$, continuous on $K$ and holomorphic in the interior of $K$, there exists a sequence of natural numbers $\left(\lambda_{n}\right)$ such that $\sum_{k=1}^{\lambda_{n}} a_{k} z^{k}$ converges to $h$ uniformly on $K$. Of course, the radius of convergence of such a power series is necessarily equal to

The present work was supported by the EPEAEK program "Pythagoras II". 
zero. Later on, universal Taylor series with strictly positive radius of convergence were defined, first independently by Luh [20] and Chui and Parnes [8] and finally, in their strongest sense, by Nestoridis [28]. Moreover, Melas and Nestoridis have studied in [24] the existence of universal Taylor series with respect to summability methods (see also $[5,7])$. Next, in [2], F. Bayart proved, among others, that if $f$ is a holomorphic function in a "suitable" simply connected domain $\Omega$, then its Taylor series is universal if and only if its Cesàro $(C, k)$-means are universal for any integer $k \geq 0$. Our aim here is to give a new proof of this result, extending this to every real $k>-1$.

Let us first recall some notations and definitions. Let $\Omega$ be a simply connected domain in the complex plane, $\Omega \neq \mathbb{C}$. We denote by $H(\Omega)$ the set of holomorphic functions on $\Omega$, endowed with the topology of uniform convergence on compact sets. For $f \in H(\Omega)$ we denote by $S_{n}(f, \xi)$ the $n t h$-partial sum of the Taylor series of $f$ with center at $\xi \in \Omega$, that is

$$
S_{n}(f, \xi)(z)=\sum_{\nu=0}^{n} a_{\nu}(z-\xi)^{\nu} \quad(z \in \mathbb{C}) .
$$

Then, for real $k>-1$, the $(C, k)$-means of order $n$ of the Taylor expansion of $f$ with center $\xi$, are given by

$$
\sigma_{n}^{k}(f, \xi)(z)=\sum_{\nu=0}^{n} \frac{A_{n-\nu}^{k}}{A_{n}^{k}} a_{\nu}(z-\xi)^{\nu}
$$

where

$$
A_{m}^{j}=\frac{\Gamma(m+j+1)}{\Gamma(m+1) \Gamma(j+1)}=\frac{(j+1)(j+2) \cdots(j+m)}{m !},
$$

i.e., $A_{m}^{j}$ equals to $\left(\begin{array}{c}m+j \\ m\end{array}\right)$, or, if $j$ is an integer, to $\left(\begin{array}{c}m+j \\ j\end{array}\right)$. The $\Gamma$ in the above formula is Euler's Gamma function. We refer to [33] for standard properties of Cesàro means of series. Further, for $K$ a subset of $\mathbb{C}$, we denote by $K^{\circ}$ its interior and by $K^{c}$ its complement. Finally, $D(w, r)$ and $\bar{D}(w, r)$ stand for the open and closed disk, respectively, with center $w \in \mathbb{C}$ and radius $r>0$.

In the following we shall prove our main results for universal Taylor series in the sense of Nestoridis. Then, the corresponding results for universal Taylor series in the sense of Luh and Chui - Parnes follow easily.

Definition 1.1. Let $f \in H(\Omega), \xi \in \Omega, j \in(-1,+\infty)$ and let $K$ be a compact set in $\mathbb{C}$ with $K \cap \Omega=\emptyset, K^{c}$ connected and $h: K \rightarrow \mathbb{C}$ be a continuous function on $K$ and holomorphic in $K^{\circ}$.

- If, for every $K$ and $h$ as above, there exists a sequence $\left(\lambda_{n}\right)$ of natural numbers such that

$$
\sup _{z \in K}\left|S_{\lambda_{n}}(f, \xi)(z)-h(z)\right| \rightarrow 0 \text {, as } n \rightarrow \infty,
$$

then we say that $f$ belongs to the class $U\left(\xi, \Omega^{c}\right)$. 
- If, for every $K$ and $h$ as above, there exists a sequence $\left(\lambda_{n}\right)$ of natural numbers such that, for every compact set $N \subset \Omega$,

$$
\sup _{\xi \in N} \sup _{z \in K}\left|S_{\lambda_{n}}(f, \xi)(z)-h(z)\right| \rightarrow 0 \text {, as } n \rightarrow \infty,
$$

then we say that $f$ belongs to the class $U\left(\Omega, \Omega^{c}\right)$.

- If, for every $K$ and $h$ as above, there exists a sequence $\left(\lambda_{n}\right)$ of natural numbers such that

$$
\sup _{z \in K}\left|\sigma_{\lambda_{n}}^{j}(f, \xi)(z)-h(z)\right| \rightarrow 0, \text { as } n \rightarrow \infty,
$$

then we say that $f$ belongs to the class $U_{C e s(j)}\left(\xi, \Omega^{c}\right)$.

- If, for every $K$ and $h$ as above, there exists a sequence $\left(\lambda_{n}\right)$ of natural numbers such that, for every compact set $N \subset \Omega$,

$$
\sup _{\xi \in N} \sup _{z \in K}\left|\sigma_{\lambda_{n}}^{j}(f, \xi)(z)-h(z)\right| \rightarrow 0 \text {, as } n \rightarrow \infty,
$$

then we say that $f$ belongs to the class $U_{C e s(j)}\left(\Omega, \Omega^{c}\right)$.

- If, for every $K$ and $h$ as above, there exists a sequence $\left(\lambda_{n}\right)$ of natural numbers such that, for every $j>-1$,

$$
\sup _{z \in K}\left|\sigma_{\lambda_{n}}^{j}(f, \xi)(z)-h(z)\right| \rightarrow 0, \text { as } n \rightarrow \infty,
$$

then we say that $f$ belongs to the class $U_{C e s}\left(\xi, \Omega^{c}\right)$.

- If, for every $K$ and $h$ as above, there exists a sequence $\left(\lambda_{n}\right)$ of natural numbers such that, for every compact set $N \subset \Omega$ and for every $j>-1$,

$$
\sup _{\xi \in N} \sup _{z \in K}\left|\sigma_{\lambda_{n}}^{j}(f, \xi)(z)-h(z)\right| \rightarrow 0 \text {, as } n \rightarrow \infty,
$$

then we say that $f$ belongs to the class $U_{C e s}\left(\Omega, \Omega^{c}\right)$.

Definition 1.2. If in Definition 1.1 the compact set $K$ does not meet the boundary of $\Omega$, i.e. $K \cap \bar{\Omega}=\emptyset$, then we have the definitions of the corresponding classes

$$
U\left(\xi, \bar{\Omega}^{c}\right), U\left(\Omega, \bar{\Omega}^{c}\right), U_{C e s(j)}\left(\xi, \bar{\Omega}^{c}\right), U_{C e s(j)}\left(\Omega, \bar{\Omega}^{c}\right), U_{C e s}\left(\xi, \bar{\Omega}^{c}\right) \text { and } U_{C e s}\left(\Omega, \bar{\Omega}^{c}\right),
$$

in the sense of Luh and Chui - Parnes.

Remark 1.3. In all the above definitions it is equivalent to require $\lambda_{n}<\lambda_{n+1}$. Furthermore, suitable applications of Baire's Theorem and Mergelyan's Theorem show that all the above classes are dense and $G_{\delta}$ subsets of $H(\Omega)([3,13,24,26,28,29])$. For the importance of generic results and the role of Baire's Theorem in Complex, Harmonic 
and Functional Analysis we refer to $[14,15]$. Properties of the universal functions belonging to these classes can be found in $[1,2,4,9-12,16,19,23-28,30,31]$. For example, if $\Omega=\mathbb{D}=D(0,1)$ we have $U\left(0, \mathbb{D}^{c}\right) \neq U\left(0, \overline{\mathbb{D}}^{c}\right)([26])$. In general the classes $U\left(\xi, \Omega^{c}\right)$ and $U\left(\Omega, \Omega^{c}\right)$ contain functions, which have wild properties; in particular they cannot be smooth on the boundary ([24], [25], [26]) and they are holomorphic exactly in $\Omega$ ([24], [31]). On the contrary the classes $U\left(\xi, \bar{\Omega}^{c}\right)$ and $U\left(\Omega, \bar{\Omega}^{c}\right)$ contain also smooth functions up to the boundary, provided that $\{\infty\} \cup \bar{\Omega}^{c}$ is connected ([25], [26], [16]), or holomorphically extendable outside $\Omega$, provided that $\partial \Omega \neq \partial \bar{\Omega}$ ([31]).

In the sequel we shall need some of the properties proved in the papers mentioned in Remark 1.3. We will present these at the beginning of Section 3. In Section 2, first we give the definition of Ostrowski gaps. Then, we prove a basic proposition, concerning subsequences of the Cesàro means of the sequence of the partial sums of a series possessing Ostrowski gaps, required for the proof of our main results. In Section 3 we prove our main results. We notice that in order to obtain these results, we shall impose some restrictions on the domain $\Omega$. More precisely, we define:

Definition 1.4. Let $\Omega$ be a simply connected domain. We shall say that $\Omega$ is admissible, if $\Omega$ is contained in the complement of an angle with strictly positive opening and it satisfies the following geometrical and topological restrictions:

For any compact set $K \subset \mathbb{C}$ with $K^{c}$ connected and such that $K \cap \Omega=\emptyset$ for the case of Definition 1.1 and $K \cap \bar{\Omega}=\emptyset$ for the case of Definition 1.2, there exist two compact subsets $L$ and $M$ of $\mathbb{C}$ and a positive real number $r$, such that $K \subset L \subset M$ and

- $M \cap \Omega=\emptyset$ for the case of Definition 1.1 and $M \cap \bar{\Omega}=\emptyset$ for the case of Definition 1.2 .

- $M^{c}$ is connected.

- For any $z \in L$, there exists $w \in L$ such that $z \in \bar{D}(w, r)$ and $\bar{D}(w, r) \subset L$.

Remark 1.5. In [2, Section 4], F. Bayart proved that circles, squares and so on, are admissible domains. Of course, for the case of Definition 1.2, any simply connected domain $\Omega$ is admissible provided that $\Omega$ is contained in the complement of an angle with strictly positive opening.

Now, the main result, which we shall prove in Section 3, is the following:

Theorem 1.6. If $\Omega$ is an admissible simply connected domain, then all the classes of universal functions in Definition 1.1 coincide and the same is true for the classes of Definition 1.2. In particular, for the classes of Definition 1.2, this is true for any simply connected domain $\Omega$ such that $\Omega$ is contained in the complement of an angle with strictly positive opening. 


\section{Ostrowski gaps and Cesàro means}

By [33, Chapter III, Theorem 1.21] we know that

if a series is summable $(C, j), j>-1$, to sum $s$, it is also summable $(C, j+\lambda)$ to $\mathrm{s}$, for every $\lambda>0$.

Moreover, if we assume that the series possesses gaps $\left(p_{m}, q_{m}\right)$ with $\frac{q_{m}}{p_{m}} \geq q>1$, which means that for $p_{m}<\nu \leq q_{m}$ its terms $a_{\nu}$ equal zero, then ([33, Chapter III, Theorem 1.27])

if it is summable $(C, 1)$ to sum $s$, the subsequence $S_{p_{m}}$ (and so $S_{q_{m}}$ too) of its partial sums tends to $s$.

In this section we shall prove that under some further restrictions, a stronger result of this kind is true for subsequences of Cesàro means of complex series possessing Ostrowski gaps (see Proposition 2.3).

We start by recalling the definition of Ostrowski gaps. Ostrowski gaps were successfully used, for example in $[2,4,6,8,21,22,24,27]$, to obtain certain properties of universal Taylor series with respect to overconvergence. Here, in order to prove our results, Ostrowski gaps will also be our main tool.

Definition 2.1. Let $\sum_{\nu=0}^{\infty} a_{\nu}$ be a series of complex numbers, or, more generally, let $\sum_{\nu=0}^{\infty} a_{\nu}(z-\xi)^{\nu}$ be a power series with positive radius of convergence. We say that each of them has Ostrowski gaps $\left(p_{m}, q_{m}\right)$, if $\left(p_{m}\right)$ and $\left(q_{m}\right)$ are two sequences of natural numbers such that:

(i) $p_{1}<q_{1} \leq p_{2}<q_{2} \leq p_{3}<q_{3} \leq \cdots$ and $\lim _{m \rightarrow \infty} \frac{q_{m}}{p_{m}}=\infty$.

(ii) For $I=\bigcup_{m=1}^{\infty}\left\{p_{m}+1, \ldots, q_{m}\right\}$, we have $\lim _{\nu \in I}\left|a_{\nu}\right|^{\frac{1}{\nu}}=0$.

Remark 2.2. By condition (ii) of Definition 2.1 we can subtract a constant from $\sum_{\nu=0}^{\infty} a_{\nu}$, or an entire function from $\sum_{\nu=0}^{\infty} a_{\nu}(z-\xi)^{\nu}$, in order to have

$$
a_{p_{m}+1}=a_{p_{m}+2}=\cdots=a_{q_{m}}=0 .
$$

Thus, without loss of generality, we shall assume this in the sequel.

Now, our basic proposition of this section is the following:

Proposition 2.3. Let $\sum_{\nu=0}^{\infty} a_{\nu}$ be a complex series with Ostrowski gaps $\left(p_{m}, q_{m}\right)$. If for some $j>-1$ we have

$$
\sigma_{q_{m}}^{j} \rightarrow s, \text { as } m \rightarrow \infty
$$

and there are constants $B \geq 2$ and $C_{1}>0$ such that

$$
\forall \nu \in \mathbb{N}, \quad\left|a_{\nu}\right| \leq B^{\nu}
$$


and

$$
\left|\sum_{\nu=k}^{p_{m}} \nu(\nu-1) \cdots(\nu-k+1) \frac{A_{q_{m}-\nu}^{j}}{A_{q_{m}^{j}}} a_{\nu}\right| \leq C_{1}\left(B p_{m}\right)^{k}, \quad k=1,2, \ldots, p_{m},
$$

then

- for $j>-1, \sigma_{q_{m}}^{j+1} \rightarrow s$ and also

- for $j \geq 0, \sigma_{q_{m}}^{j+\lambda} \rightarrow s$, for every $\lambda,-1<\lambda<1$.

In order to prove Proposition 2.3 we shall need two lemmas, which we state and prove immediately after the following remarks. First, as we notice in Remark 2.2 we can assume that

$$
a_{p_{m}+1}=a_{p_{m}+2}=\cdots=a_{q_{m}}=0 .
$$

Then, if we set $a_{n}^{m}=\frac{A_{q_{m}-n}^{j}}{A_{q_{m}}^{j}} a_{n}$ and $S_{\nu}\left(a_{n}^{m}\right)=\sum_{n=1}^{\nu} a_{n}^{m}$, we have that

$$
\sigma_{q_{m}}^{j}=a_{0}+S_{q_{m}}\left(a_{n}^{m}\right)=\cdots=a_{0}+S_{p_{m}}\left(a_{n}^{m}\right) .
$$

By (1) we conclude that there exists a constant $C_{2}>0$, such that $\left|S_{p_{m}+1}\left(a_{n}^{m}\right)\right|=$ $\left|S_{p_{m}}\left(a_{n}^{m}\right)\right| \leq C_{2}$. Let $C=\max \left\{1, C_{1}, C_{2}\right\}$. Having now the sequences $\left(p_{m}\right)$ and $\left(q_{m}\right)$, the constants $B$ and $C$ and the fact that $\frac{p_{m}}{q_{m}} \rightarrow 0$ as $m \rightarrow \infty$, we obtain that for every $\varepsilon>0$ there exists $m_{0} \in \mathbb{N}$ such that for every $m \geq m_{0}$

$$
C \frac{(B+1) p_{m}}{q_{m}-1-(B+1) p_{m}}<\frac{\varepsilon}{4}
$$

If in (4) we take $\varepsilon=1$, then the corresponding $m_{0}$ is independent of $\varepsilon$ and we denote this by $m_{1}$. In the following lemma, we shall use this $m_{1}$.

Lemma 2.4. For $m \in \mathbb{N}$ we denote by $\left(a_{n}^{m}\right)$ a sequence of complex numbers (see for example the sequence $\left(a_{n}^{m}\right)$ defined above). Let $S_{\nu}\left(a_{n}^{m}\right)=\sum_{n=1}^{\nu} a_{n}^{m}$ and let $\left(p_{m}\right),\left(q_{m}\right)$ be two sequences of natural numbers, satisfying $(i)$ of Definition 2.1. Assume also that there exist two constants $B \geq 2$ and $C \geq 1$ such that:

$$
\begin{gathered}
\forall m \geq m_{1}, \quad\left|S_{p_{m}}\left(a_{n}^{m}\right)\right| \leq C \quad \text { and }\left|S_{p_{m}+1}\left(a_{n}^{m}\right)\right| \leq C . \\
\forall m \geq m_{1}, \quad\left|a_{\nu}^{m}\right| \leq B^{\nu} \quad \text { for } \quad \nu \leq p_{m} . \\
\forall m \geq m_{1},\left|\sum_{\nu=k}^{p_{m}} \nu(\nu-1) \cdots(\nu-k+1) a_{\nu}^{m}\right| \leq C\left(B p_{m}\right)^{k}, \quad k=1,2, \ldots, p_{m} .
\end{gathered}
$$

Then we have:

$$
\forall m \geq m_{1},\left|S_{\nu}\left(a_{n}^{m}\right)\right| \leq B^{\nu+1} \text { for } \nu<p_{m}, \text { and }\left|\sum_{\nu=1}^{p_{m}-1} S_{\nu}\left(a_{n}^{m}\right)\right| \leq C(B+1) p_{m} .
$$




$$
\forall m \geq m_{1},\left|\sum_{\nu=k}^{\mu} \nu(\nu-1) \cdots(\nu-k+1) S_{\nu}\left(a_{n}^{m}\right)\right| \leq C\left(B p_{m}\right)^{k+1}, \mu=p_{m}, p_{m}+1,
$$

and if $\left(x_{m}\right)$ is a sequence of real numbers with $x_{m}>q_{m}-1$, then

$$
\forall m \geq m_{1},\left|\sum_{\nu=1}^{p_{m}-1} \frac{1}{x_{m}-\nu} S_{\nu}\left(a_{n}^{m}\right)\right| \leq C \frac{(B+1) p_{m}}{x_{m}-(B+1) p_{m}} .
$$

Moreover, for $m \in \mathbb{N}$ and $\nu \leq p_{m}+1$ we define

$$
b_{\nu}^{m}=\frac{1}{x_{m}-\nu} S_{\nu}\left(a_{n}^{m}\right) .
$$

Then, (5), (6) and (7) are valid if we replace $\left(a_{n}^{m}\right)$ by $\left(b_{n}^{m}\right)$ and thus the same is true for (8), (9) and (10) with $\left(b_{n}^{m}\right)$ instead of $\left(a_{n}^{m}\right)$.

Proof. For the proof we shall need the following well known formula of summation by parts

$$
\sum_{\nu=1}^{n} c_{\nu} u_{\nu}=\sum_{\nu=1}^{n-1}\left(c_{\nu}-c_{\nu+1}\right) S_{\nu}+c_{n} S_{n}
$$

where $S_{k}=u_{1}+\cdots+u_{k}, k=1,2, \ldots, n$. Appropriately modified, it yields $\left(c_{k}=0\right)$

$$
\sum_{\nu=k+1}^{n} c_{\nu} u_{\nu}=\sum_{\nu=k}^{n-1}\left(c_{\nu}-c_{\nu+1}\right) S_{\nu}+c_{n} S_{n}
$$

for every $k \geq 1$.

Now, in order to prove (8), we have by summation by parts that

$$
\sum_{\nu=1}^{p_{m}} \nu a_{\nu}^{m}=-\sum_{\nu=1}^{p_{m}-1} S_{\nu}\left(a_{n}^{m}\right)+p_{m} S_{p_{m}}\left(a_{n}^{m}\right) .
$$

Then, by (5) and (7) we obtain that for every $m \geq m_{1}$,

$$
\left|\sum_{\nu=1}^{p_{m}-1} S_{\nu}\left(a_{n}^{m}\right)\right| \leq C B p_{m}+C p_{m}=C(B+1) p_{m}
$$

The first part of (8) is trivial:

$$
\left|S_{\nu}\left(a_{n}^{m}\right)\right| \leq \sum_{n=1}^{\nu}\left|a_{n}^{m}\right| \leq B+B^{2}+\cdots+B^{\nu}=B \frac{B^{\nu}-1}{B-1} \leq B^{\nu+1},
$$


since $B \geq 2$. For (9), we have by summation by parts that

$$
\begin{aligned}
& \sum_{\nu=k+1}^{p_{m}} \nu(\nu-1) \cdots(\nu-k) a_{\nu}^{m} \\
& =-(k+1) \sum_{\nu=k}^{p_{m}-1} \nu(\nu-1) \cdots(\nu-k+1) S_{\nu}\left(a_{n}^{m}\right)+p_{m}\left(p_{m}-1\right) \cdots\left(p_{m}-k\right) S_{p_{m}}\left(a_{n}^{m}\right) .
\end{aligned}
$$

Then, again by (5) and (7) we obtain that for every $m \geq m_{1}$,

$$
\left|\sum_{\nu=k}^{p_{m}-1} \nu(\nu-1) \cdots(\nu-k+1) S_{\nu}\left(a_{n}^{m}\right)\right| \leq \frac{C\left(B^{k+1}+1\right)}{k+1} p_{m}^{k+1} \leq C\left(B p_{m}\right)^{k+1} .
$$

Moreover, for every $m \geq m_{1}$, it is evident that

$$
\left|\sum_{\nu=k}^{p_{m}} \nu(\nu-1) \cdots(\nu-k+1) S_{\nu}\left(a_{n}^{m}\right)\right| \leq \frac{C\left(B^{k+1}+1\right)}{k+1} p_{m}^{k+1}+C p_{m}^{k} \leq C\left(B p_{m}\right)^{k+1}
$$

To prove (10) consider a sequence of real numbers $\left(x_{m}\right)$ with $x_{m}>q_{m}-1$. Then, by the definition of $m_{1}$ (see (4)), we have that for every $m \geq m_{1}$,

$$
C \frac{(B+1) p_{m}}{x_{m}-(B+1) p_{m}} \leq C \frac{(B+1) p_{m}}{q_{m}-1-(B+1) p_{m}}<\frac{1}{4} .
$$

Besides this, we shall use frequently below the fact that

$$
\frac{B}{x_{m}-p_{m}}, \frac{C}{x_{m}-p_{m}}, \frac{C}{x_{m}-p_{m}-1} \text { and } \frac{B p_{m}}{x_{m}-p_{m}}
$$


are less than $C \frac{(B+1) p_{m}}{x_{m}-(B+1) p_{m}}$ and so less than $\frac{1}{4}$ for every $m \geq m_{1}$. Now we have

$$
\begin{aligned}
\sum_{\nu=1}^{p_{m}-1} \frac{1}{x_{m}-\nu} S_{\nu}\left(a_{n}^{m}\right)= & \frac{1}{x_{m}} \sum_{\nu=1}^{p_{m}-1} \frac{x_{m}}{x_{m}-\nu} S_{\nu}\left(a_{n}^{m}\right) \\
= & \frac{1}{x_{m}} \sum_{\nu=1}^{p_{m}-1} S_{\nu}\left(a_{n}^{m}\right)+\frac{1}{x_{m}} \sum_{\nu=1}^{p_{m}-1} \frac{\nu}{x_{m}-\nu} S_{\nu}\left(a_{n}^{m}\right) \\
= & \frac{1}{x_{m}} \sum_{\nu=1}^{p_{m}-1} S_{\nu}\left(a_{n}^{m}\right)+\frac{1}{x_{m}\left(x_{m}-1\right)} \sum_{\nu=1}^{p_{m}-1} \nu S_{\nu}\left(a_{n}^{m}\right) \\
& +\frac{1}{x_{m}\left(x_{m}-1\right)} \sum_{\nu=1}^{p_{m}-1} \frac{\nu(\nu-1)}{x_{m}-\nu} S_{\nu}\left(a_{n}^{m}\right)=\ldots \\
= & \frac{1}{x_{m}} \sum_{\nu=1}^{p_{m}-1} S_{\nu}\left(a_{n}^{m}\right)+\frac{1}{x_{m}\left(x_{m}-1\right)} \sum_{\nu=1}^{p_{m}-1} \nu S_{\nu}\left(a_{n}^{m}\right) \\
& +\frac{1}{x_{m}\left(x_{m}-1\right)\left(x_{m}-2\right)} \sum_{\nu=2}^{p_{m}-1} \nu(\nu-1) S_{\nu}\left(a_{n}^{m}\right) \\
& +\frac{1}{x_{m}\left(x_{m}-1\right) \cdots\left(x_{m}-3\right)} \sum_{\nu=3}^{p_{m}-1} \nu(\nu-1)(\nu-2) S_{\nu}\left(a_{n}^{m}\right)+\ldots \\
& +\frac{\left(p_{m}-1\right) !}{x_{m}\left(x_{m}-1\right) \cdots\left(x_{m}-p_{m}+1\right)} S_{p_{m}-1}\left(a_{n}^{m}\right) .
\end{aligned}
$$

Thus, by (8) and (9) we easily obtain that

$$
\begin{aligned}
\left|\sum_{\nu=1}^{p_{m}-1} \frac{1}{x_{m}-\nu} S_{\nu}\left(a_{n}^{m}\right)\right| & \leq C \frac{(B+1) p_{m}}{x_{m}-p_{m}}\left(1+\frac{B p_{m}}{x_{m}-p_{m}}+\left(\frac{B p_{m}}{x_{m}-p_{m}}\right)^{2}+\cdots\right) \\
& =C \frac{(B+1) p_{m}}{x_{m}-(B+1) p_{m}} .
\end{aligned}
$$

It remains to prove (5), (6) and (7) for the sequence $\left(b_{n}^{m}\right)$. Since

$$
b_{\nu}^{m}=\frac{1}{x_{m}-\nu} S_{\nu}\left(a_{n}^{m}\right),
$$

we have by (5) and (10) that for every $m \geq m_{1}$

$$
\begin{aligned}
\left|S_{p_{m}}\left(b_{n}^{m}\right)\right| & =\left|\sum_{\nu=1}^{p_{m}} b_{\nu}^{m}\right| \leq\left|\sum_{\nu=1}^{p_{m}-1} \frac{1}{x_{m}-\nu} S_{\nu}\left(a_{n}^{m}\right)\right|+\left|\frac{1}{x_{m}-p_{m}} S_{p_{m}}\left(a_{n}^{m}\right)\right| \\
& \leq C \frac{(B+1) p_{m}}{x_{m}-(B+1) p_{m}}+\frac{C}{x_{m}-p_{m}} \leq C,
\end{aligned}
$$


and similarly

$$
\left|S_{p_{m}+1}\left(b_{n}^{m}\right)\right| \leq C \frac{(B+1) p_{m}}{x_{m}-(B+1) p_{m}}+\frac{C}{x_{m}-p_{m}}+\frac{C}{x_{m}-p_{m}-1} \leq C .
$$

Furthermore, by (8)

$$
\left|b_{\nu}^{m}\right|=\left|\frac{1}{x_{m}-\nu} S_{\nu}\left(a_{n}^{m}\right)\right| \leq \frac{B}{x_{m}-p_{m}} B^{\nu} \leq B^{\nu} .
$$

Finally,

$$
\begin{aligned}
\sum_{\nu=k}^{p_{m}} \nu(\nu-1) \cdots(\nu-k+1) b_{\nu}^{m} & =\sum_{\nu=k}^{p_{m}} \nu(\nu-1) \cdots(\nu-k+1) \frac{1}{x_{m}-\nu} S_{\nu}\left(a_{n}^{m}\right) \\
& =\frac{1}{x_{m}-k} \sum_{\nu=k}^{p_{m}} \nu(\nu-1) \cdots(\nu-k+1) \frac{x_{m}-k}{x_{m}-\nu} S_{\nu}\left(a_{n}^{m}\right),
\end{aligned}
$$

and continuing as in the proof of (10), we arrive at the following formula:

$$
\begin{aligned}
& \sum_{\nu=k}^{p_{m}} \nu(\nu-1) \cdots(\nu-k+1) b_{\nu}^{m}=\frac{1}{x_{m}-k} \sum_{\nu=k}^{p_{m}} \nu(\nu-1) \cdots(\nu-k+1) S_{\nu}\left(a_{n}^{m}\right) \\
& +\frac{1}{\left(x_{m}-k\right)\left(x_{m}-k-1\right)} \sum_{\nu=k+1}^{p_{m}} \nu(\nu-1) \cdots(\nu-k) S_{\nu}\left(a_{n}^{m}\right)+\ldots \\
& \quad+\frac{p_{m} !}{\left(x_{m}-k\right)\left(x_{m}-k-1\right) \cdots\left(x_{m}-p_{m}\right)} S_{p_{m}}\left(a_{n}^{m}\right) .
\end{aligned}
$$

Then, by (5) and (9) we conclude that for every $m \geq m_{1}$ we have

$$
\begin{aligned}
\left|\sum_{\nu=k}^{p_{m}} \nu(\nu-1) \cdots(\nu-k+1) b_{\nu}^{m}\right| & \leq C\left(B p_{m}\right)^{k}\left(\frac{B p_{m}}{x_{m}-p_{m}}+\left(\frac{B p_{m}}{x_{m}-p_{m}}\right)^{2}+\cdots\right) \\
& =C\left(B p_{m}\right)^{k} \frac{B p_{m}}{x_{m}-(B+1) p_{m}} \leq C\left(B p_{m}\right)^{k},
\end{aligned}
$$

and the proof is complete.

From Lemma 2.4 we conclude that everything we can prove for the sequence $\left(a_{n}^{m}\right)$, using (5) to (10), is automatically true for the sequence $\left(b_{n}^{m}\right)$. For example, by summation by parts we have:

$$
\begin{aligned}
& \sum_{\nu=1}^{p_{m}}\left(\frac{1}{x_{m}}+\frac{1}{x_{m}-1}+\cdots+\frac{1}{x_{m}-\nu+1}\right) a_{\nu}^{m}=-\sum_{\nu=1}^{p_{m}-1} \frac{1}{x_{m}-\nu} S_{\nu}\left(a_{n}^{m}\right) \\
& \quad+\left(\frac{1}{x_{m}}+\frac{1}{x_{m}-1}+\cdots+\frac{1}{x_{m}-p_{m}+1}\right) S_{p_{m}}\left(a_{n}^{m}\right) .
\end{aligned}
$$


So, by (5) and (10) we have

$$
\left|\sum_{\nu=1}^{p_{m}}\left(\frac{1}{x_{m}}+\frac{1}{x_{m}-1}+\cdots+\frac{1}{x_{m}-\nu+1}\right) a_{\nu}^{m}\right| \leq 2 C \frac{(B+1) p_{m}}{x_{m}-(B+1) p_{m}},
$$

for $m \geq m_{1}$. Since $x_{m}>q_{m}-1$, we have by (4) that for every $\varepsilon \in(0,1)$ the corresponding $m_{0}$ is greater than $m_{1}$ and so for every $m \geq m_{0}$ we have

$$
\left|\sum_{\nu=1}^{p_{m}}\left(\frac{1}{x_{m}}+\frac{1}{x_{m}-1}+\cdots+\frac{1}{x_{m}-\nu+1}\right) a_{\nu}^{m}\right|<\frac{\varepsilon}{2} .
$$

Then, we automatically conclude that for the same $m \geq m_{0}$ it is also true that

$$
\left|\sum_{\nu=1}^{p_{m}}\left(\frac{1}{x_{m}}+\frac{1}{x_{m}-1}+\cdots+\frac{1}{x_{m}-\nu+1}\right) b_{\nu}^{m}\right|<\frac{\varepsilon}{2} .
$$

Let us suppose now that for the sequence $\left(a_{n}^{m}\right)$ of Lemma 2.4 we have that $a_{p_{m}+1}^{m}$ tends to zero and more precisely

$$
\left|a_{p_{m}+1}^{m}\right| \leq \frac{C}{x_{m}-p_{m}-1},
$$

in accordance to $b_{p_{m}+1}^{m}$ (in fact we shall take later $a_{p_{m}+1}^{m}=0$ ). Next, we denote by

$$
s_{k}\left(\frac{1}{x_{m}}, \frac{1}{x_{m}-1}, \cdots, \frac{1}{x_{m}-n}\right)=\sum_{0 \leq j_{1}<\cdots<j_{k} \leq n} \frac{1}{\left(x_{m}-j_{1}\right) \cdots\left(x_{m}-j_{k}\right)},
$$

$k=2,3, \ldots, n$, the kth symmetric polynomial in the variables $\frac{1}{x_{m}}, \frac{1}{x_{m}-1}, \cdots, \frac{1}{x_{m}-n}$. Then, by summation by parts we have

$$
\begin{aligned}
\sum_{\nu=2}^{p_{m}+1} s_{2} & \left(\frac{1}{x_{m}}, \frac{1}{x_{m}-1}, \cdots, \frac{1}{x_{m}-\nu+1}\right) a_{\nu}^{m} \\
= & -\sum_{\nu=1}^{p_{m}}\left(\frac{1}{x_{m}}+\frac{1}{x_{m}-1}+\cdots+\frac{1}{x_{m}-\nu+1}\right) \frac{1}{x_{m}-\nu} S_{\nu}\left(a_{n}^{m}\right) \\
& +s_{2}\left(\frac{1}{x_{m}}, \frac{1}{x_{m}-1}, \cdots, \frac{1}{x_{m}-p_{m}}\right) S_{p_{m}+1}\left(a_{n}^{m}\right) \\
= & -\sum_{\nu=1}^{p_{m}}\left(\frac{1}{x_{m}}+\frac{1}{x_{m}-1}+\cdots+\frac{1}{x_{m}-\nu+1}\right) b_{\nu}^{m} \\
& +s_{2}\left(\frac{1}{x_{m}}, \frac{1}{x_{m}-1}, \cdots, \frac{1}{x_{m}-p_{m}}\right) S_{p_{m}+1}\left(a_{n}^{m}\right) .
\end{aligned}
$$


which, by (5) and (11), implies that

$$
\left|\sum_{\nu=2}^{p_{m}+1} s_{2}\left(\frac{1}{x_{m}}, \frac{1}{x_{m}-1}, \cdots, \frac{1}{x_{m}-\nu+1}\right) a_{\nu}^{m}\right| \leq \frac{\varepsilon}{2}+\frac{C}{2}\left(\frac{p_{m}+1}{x_{m}-p_{m}}\right)^{2}
$$

and so

$$
\begin{aligned}
\left|\sum_{\nu=2}^{p_{m}} s_{2}\left(\frac{1}{x_{m}}, \frac{1}{x_{m}-1}, \cdots, \frac{1}{x_{m}-\nu+1}\right) a_{\nu}^{m}\right| & \leq \frac{\varepsilon}{2}+\frac{C}{2}\left(\frac{p_{m}+1}{x_{m}-p_{m}}\right)^{2}+\frac{C p_{m}\left(p_{m}+1\right)}{2\left(x_{m}-p_{m}\right)^{3}} \\
& \leq \frac{\varepsilon}{2}+\left(\frac{\varepsilon}{2}\right)^{2}
\end{aligned}
$$

since

$$
\begin{aligned}
\frac{C}{2}\left(\frac{p_{m}+1}{x_{m}-p_{m}}\right)^{2}+\frac{C p_{m}\left(p_{m}+1\right)}{2\left(x_{m}-p_{m}\right)^{3}} & \leq \frac{C}{2}\left(\frac{p_{m}+1}{x_{m}-p_{m}}\right)^{2}\left(1+\frac{1}{x_{m}-p_{m}}\right) \\
& \leq\left(2 C \frac{(B+1) p_{m}}{x_{m}-(B+1) p_{m}}\right)^{2} \leq\left(\frac{\varepsilon}{2}\right)^{2}
\end{aligned}
$$

for every $m \geq m_{0}$. Then, we automatically conclude that for the same $m \geq m_{0}$ it is also true that

$$
\left|\sum_{\nu=2}^{p_{m}} s_{2}\left(\frac{1}{x_{m}}, \frac{1}{x_{m}-1}, \cdots, \frac{1}{x_{m}-\nu+1}\right) b_{\nu}^{m}\right| \leq \frac{\varepsilon}{2}+\left(\frac{\varepsilon}{2}\right)^{2},
$$

which we shall need in order to continue one more step further. Thus, for every $m \geq m_{0}$, an easy induction argument on $k$ implies that the following holds:

$$
\left|\sum_{\nu=k}^{p_{m}} s_{k}\left(\frac{1}{x_{m}}, \frac{1}{x_{m}-1}, \cdots, \frac{1}{x_{m}-\nu+1}\right) a_{\nu}^{m}\right| \leq \sum_{n=1}^{k}\left(\frac{\varepsilon}{2}\right)^{n} .
$$

Since for every $\varepsilon<1, \sum_{n=1}^{k}\left(\frac{\varepsilon}{2}\right)^{n}<\varepsilon$, we have proved the following:

Lemma 2.5. If for the sequence $\left(a_{n}^{m}\right)$ of Lemma 2.4 we have that $a_{p_{m}+1}^{m}=0$, then for every $0<\varepsilon<1$ there exists $m_{0} \in \mathbb{N}$ such that for all $m \geq m_{0}$

$$
\left|\sum_{\nu=k}^{p_{m}} s_{k}\left(\frac{1}{x_{m}}, \frac{1}{x_{m}-1}, \cdots, \frac{1}{x_{m}-\nu+1}\right) a_{\nu}^{m}\right|<\varepsilon,
$$

$k=1,2, \ldots, p_{m}$.

We are now ready to prove our basic Proposition 2.3. 
Proof. Without loss of generality, we assume that $a_{p_{m}+1}=\cdots=a_{q_{m}}=0$. Thus,

$$
\begin{gathered}
\sigma_{q_{m}}^{j+\lambda}=\sum_{\nu=0}^{q_{m}} \frac{A_{q_{m}-\nu}^{j+\lambda}}{A_{q_{m}}^{j+\lambda}} a_{\nu}=\sum_{\nu=0}^{p_{m}} \frac{A_{q_{m}-\nu}^{j+\lambda}}{A_{q_{m}}^{j+\lambda}} a_{\nu}= \\
=a_{0}+\sum_{\nu=1}^{p_{m}} \frac{\left(q_{m}+j\right)\left(q_{m}+j-1\right) \cdots\left(q_{m}+j-\nu+1\right)}{\left(q_{m}+j+\lambda\right) \cdots\left(q_{m}+j+\lambda-\nu+1\right)} \frac{A_{q_{m}-\nu}^{j}}{A_{q_{m}}^{j}} a_{\nu} .
\end{gathered}
$$

For $\lambda=1$ this yields that

$$
\sigma_{q_{m}}^{j+1}=a_{0}+\sum_{\nu=1}^{p_{m}} \frac{q_{m}+j-\nu+1}{q_{m}+j+1} \frac{A_{q_{m}-\nu}^{j}}{A_{q_{m}}^{j}} a_{\nu}=\sigma_{q_{m}}^{j}-\frac{1}{q_{m}+j+1} \sum_{\nu=1}^{p_{m}} \nu \frac{A_{q_{m}-\nu}^{j}}{A_{q_{m}}^{j}} a_{\nu},
$$

which implies the desired result for any $j>-1$, i.e.

$$
\left|\sigma_{q_{m}}^{j+1}-s\right| \leq\left|\sigma_{q_{m}}^{j}-s\right|+\left|\frac{1}{q_{m}+j+1} \sum_{\nu=1}^{p_{m}} \nu \frac{A_{q_{m}-\nu}^{j}}{A_{q_{m}}^{j}} a_{\nu}\right| \rightarrow 0,
$$

by (1), (3) and the fact that $\frac{p_{m}}{q_{m}} \rightarrow 0$.

For $j \geq 0$ and $-1<\lambda<1$, we set $a_{\nu}^{m}=\frac{A_{q_{m}-\nu}^{j}}{A_{q_{m}}^{j}} a_{\nu}, x_{m}=q_{m}+j+\lambda>q_{m}-1$ and we have:

$$
\begin{aligned}
\sigma_{q_{m}}^{j+\lambda}= & a_{0}+\sum_{\nu=1}^{p_{m}}\left(1-\frac{\lambda}{x_{m}}\right) \cdots\left(1-\frac{\lambda}{x_{m}-\nu+1}\right) a_{\nu}^{m} \\
= & \sigma_{q_{m}}^{j}-\lambda \sum_{\nu=1}^{p_{m}}\left(\frac{1}{x_{m}}+\frac{1}{x_{m}-1}+\cdots+\frac{1}{x_{m}-\nu+1}\right) a_{\nu}^{m} \\
& +\lambda^{2} \sum_{\nu=2}^{p_{m}} s_{2}\left(\frac{1}{x_{m}}, \frac{1}{x_{m}-1}, \cdots, \frac{1}{x_{m}-\nu+1}\right) a_{\nu}^{m} \\
& -\lambda^{3} \sum_{\nu=3}^{p_{m}} s_{3}\left(\frac{1}{x_{m}}, \frac{1}{x_{m}-1}, \cdots, \frac{1}{x_{m}-\nu+1}\right) a_{\nu}^{m}+\ldots \\
& +(-1)^{p_{m}} \lambda^{p_{m}} \frac{1}{x_{m} \cdots\left(x_{m}-p_{m}+1\right)} a_{p_{m}}^{m} .
\end{aligned}
$$

By [33, Chapter III, Theorem 1.17], we know that

$A_{n}^{j}$ is positive for $j>-1$, increasing (as a function of $n$ ) for $j \geq 0$ and decreasing for $-1<j<0$,

so that, in our case $(j \geq 0), \forall m \in \mathbb{N},\left|a_{\nu}^{m}\right| \leq\left|a_{\nu}\right| \leq B^{\nu}$ for $\nu \leq p_{m}$, by (2). Moreover, as we already saw, there exists, by (1), a constant $C_{2}>0$, such that 
$\left|S_{p_{m}+1}\left(a_{n}^{m}\right)\right|=\left|S_{p_{m}}\left(a_{n}^{m}\right)\right| \leq C_{2}$. If $C=\max \left\{1, C_{1}, C_{2}\right\}$, we see that $\left(a_{n}^{m}\right)$ satisfies the hypotheses of Lemma 2.5 and so for every $\varepsilon \in(0,1)$, there exists $m_{0} \in \mathbb{N}$ such that, for every $m \geq m_{0}$ we have that

$$
\left|\sigma_{q_{m}}^{j+\lambda}-\sigma_{q_{m}}^{j}\right|<\left(|\lambda|+|\lambda|^{2}+\cdots+|\lambda|^{p_{m}}\right) \varepsilon \leq \frac{|\lambda|}{1-|\lambda|} \varepsilon,
$$

which implies the desired result and the proof is finished.

\section{Main Results}

Now we are ready to prove our main results. First, as we mentioned in the Introduction, we recall some known properties of universal functions. In [12] it has been proved that if $\Omega$ is a bounded simply connected domain, $\xi \in \Omega$ and $f \in U\left(\xi, \bar{\Omega}^{c}\right)$, then the Taylor series of $f$ at $\xi$ has Ostrowski gaps and $U\left(\xi, \bar{\Omega}^{c}\right)=U\left(\Omega, \bar{\Omega}^{c}\right)$. This in turn is a corollary of Theorem 9.1 of [24], where, moreover, it has been proved that $U\left(\xi, \Omega^{c}\right)=U\left(\Omega, \Omega^{c}\right)$ provided that $\Omega$ is contained in the complement of an angle with strictly positive opening. Finally, the general case has been proved in [27] and we state this in the following lemma.

Lemma 3.1. Let $\Omega$ be any simply connected domain, $\xi \in \Omega$ and $f \in U\left(\xi, \Omega^{c}\right)$. Then

- The Taylor series of $f$ at $\xi$ has Ostrowski gaps.

- $U\left(\zeta, \Omega^{c}\right)=U\left(\Omega, \Omega^{c}\right)$, for any $\zeta \in \Omega$.

However, for the case of $U\left(\xi, \bar{\Omega}^{c}\right)$ we have not such a result for all simply connected domains, but we have for example the following lemma $([24,27])$.

Lemma 3.2. Let $\Omega$ be a simply connected domain such that $\bar{\Omega}^{c}$ has an unbounded component, $\xi \in \Omega$ and $f \in U\left(\xi, \bar{\Omega}^{c}\right)$. Then

- The Taylor series of $f$ at $\xi$ has Ostrowski gaps.

- $U\left(\zeta, \bar{\Omega}^{c}\right)=U\left(\Omega, \bar{\Omega}^{c}\right)$, for any $\zeta \in \Omega$.

Next, the following property for $(C, j)$-means, $j \geq 0$ an integer, has been proved in Lemma 2.6 of [2], which we state here for admissible simply connected domains $\Omega$ and $j>-1$. Thus, in our case, $j$ is no longer an integer (in general), but the proof remains unchanged, except in its last step, where instead of $j$ it suffices to use the integral part of $j$ and more precisely the integer $[j]+1$.

Lemma 3.3. Let $\Omega$ be an admissible simply connected domain, $\xi \in \Omega, j>-1$ and $f \in U_{C e s(j)}\left(\xi, \Omega^{c}\right)$. If $K$ and $h$ are as in Definition 1.1, then we have

- The Taylor series of $f$ at $\xi$ has Ostrowski gaps $\left(p_{m}, q_{m}\right)$. 
- $\sup _{z \in K}\left|\sigma_{q_{m}}^{j}(f, \xi)(z)-h(z)\right| \rightarrow 0$, as $m \rightarrow \infty$.

Finally, we will need a classical lemma which comes from Harmonic Analysis.

Lemma 3.4 ([2, Lemma 4.2]). Let $p(z)$ be a holomorphic polynomial of degree $N$, $w \in \mathbb{C}, r>0$ and $k \geq 1$. Then we have

$$
\sup _{z \in \bar{D}(w, r)}\left|p^{(k)}(z)\right| \leq \frac{N(N-1) \cdots(N-k+1)}{r^{k}} \sup _{z \in \bar{D}(w, r)}|p(z)| .
$$

For $w=0$ and $r=1$ this is just Bernstein's inequality. The general case follows easily.

We are ready now to state and prove our main Theorem.

Theorem 3.5. Let $\Omega$ be an admissible simply connected domain, $\xi \in \Omega, j>-1$ and $f \in U_{\operatorname{Ces}(j)}\left(\xi, \Omega^{c}\right)$. Then, for every compact set $K$ as in Definition 1.1 and any holomorphic polynomial $P(z)$, there exists a sequence $\left(q_{m}\right)$ of natural numbers such that, for every $\alpha>-1$,

$$
\sup _{z \in K}\left|\sigma_{q_{m}}^{\alpha}(f, \xi)(z)-P(z)\right| \rightarrow 0, \quad \text { as } m \rightarrow \infty
$$

Proof. Let $K$ be a compact set such that $K \cap \Omega=\emptyset, K^{c}$ connected and let $P(z)$ be a holomorphic polynomial. Since $\Omega$ is an admissible simply connected domain there exist compact sets $L, M$ and a positive number $r$ such that $K \subset L \subset M$, $M \cap \Omega=\emptyset, M^{c}$ connected and for any $z \in L$, there exists $w \in L$ such that $z \in \bar{D}(w, r)$ and $\bar{D}(w, r) \subset L$. By Lemma 3.3, $f \in U_{C e s(j)}\left(\xi, \Omega^{c}\right)$ implies that the Taylor series $\sum_{\nu=0}^{\infty} a_{\nu}(z-\xi)^{\nu}$ of $f$ at $\xi$ has Ostrowski gaps $\left(p_{m}, q_{m}\right)$ and

$$
\sup _{z \in M}\left|\sigma_{q_{m}}^{j}(f, \xi)(z)-P(z)\right| \rightarrow 0, \quad \text { as } m \rightarrow \infty \text {. }
$$

Hence, there exists a constant $C_{1}>0$ such that, for any $m \geq 0$, we have

$$
\sup _{w \in L}\left|\sigma_{q_{m}}^{j}(f, \xi)(w)\right| \leq C_{1}
$$

By Remark 2.2 we assume, without loss of generality, that

$$
a_{p_{m}+1}=a_{p_{m}+2}=\cdots=a_{q_{m}}=0 .
$$

Then, for every $m \geq 0$, we obtain that

$$
\sup _{w \in L}\left|\sigma_{q_{m}}^{j}(f, \xi)(w)\right|=\sup _{w \in L}\left|\sum_{\nu=0}^{p_{m}} a_{\nu}^{m}\right|=\sup _{w \in L}\left|\sum_{\nu=0}^{p_{m}+1} a_{\nu}^{m}\right| \leq C_{1},
$$


where $a_{\nu}^{m}=\frac{A_{q_{m-\nu}}^{j}}{A_{q m}^{j}} a_{\nu}(z-\xi)^{\nu}$. Since for any $z \in K$ there exists $w \in L$ such that $z \in \bar{D}(w, r)$ and $\bar{D}(w, r) \subset L$, one obtains, by Lemma 3.4 , that

$$
\left|\sigma_{q_{m}}^{j}\left(f^{(k)}, \xi\right)(z)\right| \leq \frac{p_{m} \cdots\left(p_{m}-k+1\right)}{r^{k}} C_{1}, \quad 1 \leq k \leq p_{m} .
$$

This in turn implies that for all $m \geq 0$

$$
\sup _{z \in K}\left|\sum_{\nu=k}^{p_{m}} \nu(\nu-1) \cdots(\nu-k+1) a_{\nu}^{m}\right|=\sup _{z \in K}|z-\xi|^{k}\left|\sigma_{q_{m}}^{j}\left(f^{(k)}, \xi\right)(z)\right| \leq C_{1}\left(\frac{A p_{m}}{r}\right)^{k},
$$

where $A=\sup _{z \in K}|z-\xi|$. Moreover, if $R_{\xi}$ is the radius of convergence of the Taylor series of $f$ at $\xi$ and $0<R_{1}<R_{\xi}$, then there exists $n_{0} \in \mathbb{N}$ such that $\left|a_{\nu}\right| \leq\left(\frac{1}{R_{1}}\right)^{\nu}$, for every $\nu \geq n_{0}$. So, we can find a positive number $r_{1}$ such that $\left|a_{\nu}\right| \leq\left(\frac{1}{r_{1}}\right)^{\nu}$, for all $\nu \in \mathbb{N}$. Hence, for every $\nu \in \mathbb{N}$ we have that $\left|a_{\nu}(z-\xi)^{\nu}\right| \leq\left(\frac{A}{r_{1}}\right)^{\nu}$. Taking $B=\max \left\{2, \frac{A}{r}, \frac{A}{r_{1}}\right\}$ all the hypotheses of Proposition 2.3 are fulfilled and since by (4) it is obvious that $m_{0}$ is independent from $z$, we conclude that

(i) if $j \geq 0$, then for every $\lambda \in(-1,1]$

$$
\sup _{z \in K}\left|\sigma_{q_{m}}^{j+\lambda}(f, \xi)(z)-P(z)\right| \rightarrow 0, \quad \text { as } m \rightarrow \infty,
$$

and continuing in the same way we obtain that for every $\alpha>-1$

$$
\sup _{z \in K}\left|\sigma_{q_{m}}^{\alpha}(f, \xi)(z)-P(z)\right| \rightarrow 0, \quad \text { as } m \rightarrow \infty .
$$

(ii) If $-1<j<0$, then we know (by Proposition 2.3 again) that

$$
\sup _{z \in K}\left|\sigma_{q_{m}}^{j+1}(f, \xi)(z)-P(z)\right| \rightarrow 0, \quad \text { as } m \rightarrow \infty,
$$

and since $j+1>0$ we have the required result by case (i) and the proof is finished.

Now, let $\Omega$ be an admissible simply connected domain and $f \in U_{C e s(j)}\left(\xi, \Omega^{c}\right)$. Let $K$ be a compact subset of $\mathbb{C}$ with $K \cap \Omega=\emptyset$ and $K^{c}$ connected, and let $h: K \rightarrow \mathbb{C}$ be a continuous function on $K$ and holomorphic in $K^{\circ}$. By Mergelyan's Theorem, there exists a sequence $\left(P_{n}\right)$ of holomorphic polynomials such that $P_{n} \rightarrow h$ uniformly on $K$. By Theorem 3.5, for any $n \in \mathbb{N}$, there exists a sequence $\left(q_{m}(n)\right)_{m \geq 0}$ such that, for any $\alpha>-1$, one has

$$
\sup _{z \in K}\left|\sigma_{q_{m}(n)}^{\alpha}(f, \xi)(z)-P_{n}(z)\right| \rightarrow 0, \quad \text { as } m \rightarrow \infty .
$$


Using a diagonal argument, we easily deduce that $f \in U_{C e s}\left(\xi, \Omega^{c}\right)$. In particular $f \in U\left(\xi, \Omega^{c}\right)$ and hence, by Lemma 3.1, $f \in U\left(\Omega, \Omega^{c}\right)$. Moreover, if $N$ is any compact subset of $\Omega$, we can take the positive number $R_{1}$, appearing in the proof of Theorem 3.5 , so that $0<R_{1}<\operatorname{dist}\left(N, \Omega^{c}\right)$. Then, we can repeat the proof of Theorem 3.5 and the above argument in order to deduce that, for any $\alpha>-1$ and any compact set $N \subset \Omega$, we have

$$
\sup _{\xi \in N} \sup _{z \in K}\left|\sigma_{r_{m}}^{\alpha}(f, \xi)(z)-h(z)\right| \rightarrow 0, \quad \text { as } m \rightarrow \infty,
$$

for some sequence $\left(r_{m}\right)$ of natural numbers i.e. $f \in U_{C e s}\left(\Omega, \Omega^{c}\right)$. Thus,

$$
U_{C e s(j)}\left(\xi, \Omega^{c}\right) \subset U_{C e s}\left(\Omega, \Omega^{c}\right)
$$

and since

$$
U_{C e s}\left(\Omega, \Omega^{c}\right) \subset U_{C e s(j)}\left(\Omega, \Omega^{c}\right) \subset U_{C e s(j)}\left(\xi, \Omega^{c}\right)
$$

and

$$
U_{C e s}\left(\Omega, \Omega^{c}\right) \subset U_{C e s}\left(\xi, \Omega^{c}\right) \subset U_{C e s(j)}\left(\xi, \Omega^{c}\right),
$$

we have proved the following.

Corollary 3.6. If $\Omega$ is an admissible simply connected domain, then all the classes of universal functions in Definition 1.1 coincide.

For the classes of Definition 1.2 we know, by Remark 1.5, that any simply connected domain $\Omega$, which is contained in the complement of an angle with strictly positive opening, is an admissible domain and thus, essentially the same argument as above yields the following.

Corollary 3.7. If $\Omega$ is a simply connected domain and $\Omega$ is contained in the complement of an angle with strictly positive opening, then all the classes of universal functions in Definition 1.2 coincide.

Remark 3.8. We notice that for a function $f$ holomorphic in a simply connected domain $\Omega$, there is another natural way to obtain an expansion in series: the Faber series expansion. For the existence of Universal Faber series and their properties we refer to $[4,18,22]$. In summary, let $\Gamma$ be a compact, connected subset of $\Omega$, with more than one point and $\mathbb{C} \backslash \Gamma$ connected. Let $Y$ be the set of all such subsets $\Gamma$ of $\Omega$. If $\Gamma \in Y$ and $K, h$ are as in Definition 1.1, then we say that a holomorphic function $f \in H(\Omega)$ belongs to the class $U(\Omega, \Gamma)$, if there exists a sequence $\left(\lambda_{n}\right)$ of natural numbers such that the subsequence $S_{\lambda_{n}}(f, \Gamma)(z)$ of the partial sums of the Faber series of $f$, converges to $h(z)$ uniformly on $K$. On the other hand we say that $f$ belongs to the class $U_{F a b}(\Omega)$, if for any compact set $M \subset Y$, where $Y$ is endowed with the metric topology introduced in [3], section B.5 (see also [4]), we have that

$$
\sup _{\Gamma \in M} \sup _{z \in K}\left|S_{\lambda_{n}}(f, \Gamma)(z)-h(z)\right| \rightarrow 0, \quad \text { as } n \rightarrow \infty .
$$


In [22], Mayenberger and Müller have proved that for any $\Gamma \in Y, U(\Omega, \Gamma)=U\left(\Omega, \Omega^{c}\right)$. Moreover, Theorem 1.5 in [4] extends this result to the following: For any $\Gamma \in Y$, $U(\Omega, \Gamma)=U\left(\Omega, \Omega^{c}\right)=U_{F a b}(\Omega)$. Thus, for the case of an admissible simply connected domain $\Omega$ we have that all the classes of Universal functions of Definition 1.1 coincide with the above classes of Universal Faber series. However, we do not know what happens for non-admissible domains. As pointed out to us by V. Vlachou, we can prove Lemma 3.3 using the methods of [27], in order to avoid the restriction that $\Omega$ is contained in the complement of an angle with strictly positive opening, but the general case remains still open.

Acknowledgement. I would like to thank Vasilis Nestoridis for suggesting this problem and for his comments and encouragement. I would also like to thank George Costakis and V. Vlachou for several helpful discussions and remarks.

\section{References}

[1] D. H. Armitage and G. Costakis, Boundary behavior of universal Taylor series and their derivatives, Constr. Approx. 24 (2006), no. 1, 1-15.

[2] F. Bayart, Boundary behavior and Cesàro means of universal Taylor series, Rev. Mat. Complut. 19 (2006), no. 1, 235-247.

[3] F. Bayart, K.-G. Grosse-Erdmann, V. Nestoridis, and C. Papadimitropoulos, Abstract theory of universal series and applications, Proc. Lond. Math. Soc. (3) 96 (2008), no. 2, 417-463.

[4] F. Bayart and V. Nestoridis, Universal Taylor series have a strong form of universality, J. Anal. Math. 104 (2008), 69-82.

[5] L. Bernal-González, M. C. Calderón-Moreno, and W. Luh, Universal transforms of the geometric series under generalized Riesz methods, Comput. Methods Funct. Theory 3 (2003), no. 1-2, 285297.

[6] L. Bernal-González, M. C. Calderón-Moreno, and W. Luh, Universal matrix-overconvergence of noncontinuable holomorphic functions, Anal. Math. 31 (2005), no. 4, 235-250 (English, with English and Russian summaries).

[7] Universal matrix transforms of holomorphic functions, Houston J. Math. 32 (2006), no. 1, 315-324 (electronic).

[8] C. K. Chui and M. N. Parnes, Approximation by overconvergence of a power series, J. Math. Anal. Appl. 36 (1971), 693-696.

[9] G. Costakis, Some remarks on universal functions and Taylor series, Math. Proc. Cambridge Philos. Soc. 128 (2000), no. 1, 157-175.

[10] G. Costakis, On the radial behavior of universal Taylor series, Monatsh. Math. 145 (2005), no. $1,11-17$.

[11] G. Costakis and A. Melas, On the range of universal functions, Bull. London Math. Soc. 32 (2000), no. 4, 458-464.

[12] W. Gehlen, W. Luh, and J. Müller, On the existence of O-universal functions, Complex Variables Theory Appl. 41 (2000), no. 1, 81-90.

[13] K.-G. Große-Erdmann, Holomorphe Monster und universelle Funktionen, Mitt. Math. Sem. Giessen 176 (1987), iv+84 (German). Dissertation, University of Trier, Trier, 1987. 
[14] K.-G. Grosse-Erdmann, Universal families and hypercyclic operators, Bull. Amer. Math. Soc. (N.S.) 36 (1999), no. 3, 345-381.

[15] J.-P. Kahane, Baire's category theorem and trigonometric series, J. Anal. Math. 80 (2000), 143-182.

[16] Ch. Kariofillis, Ch. Konstadilaki, and V. Nestoridis, Smooth universal Taylor series, Monatsh. Math. 147 (2006), no. 3, 249-257.

[17] C. Kariofillis and V. Nestoridis, Universal Taylor series in simply connected domains, Comput. Methods Funct. Theory 6 (2006), no. 2, 437-446.

[18] E. Katsoprinakis, V. Nestoridis, and I. Papadoperakis, Universal Faber series, Analysis (Munich) 21 (2001), no. 4, 339-363.

[19] E. S. Katsoprinakis and M. Papadimitrakis, Extensions of a theorem of Marcinkiewicz-Zygmund and of Rogosinski's formula and an application to universal Taylor series, Proc. Amer. Math. Soc. 127 (1999), no. 7, 2083-2090.

[20] W. Luh, Approximation analytischer Funktionen durch überkonvergente Potenzreihen und deren Matrix-Transformierten, Mitt. Math. Sem. Giessen Heft 88 (1970), i+56 (German).

[21] _ Universal approximation properties of overconvergent power series on open sets, Analysis 6 (1986), no. 2-3, 191-207.

[22] D. Mayenberger and J. Müller, Faber series with Ostrowski gaps, Complex Var. Theory Appl. 50 (2005), no. 2, 79-88.

[23] A. D. Melas, On the growth of universal functions, J. Anal. Math. 82 (2000), 1-20.

[24] A. Melas and V. Nestoridis, Universality of Taylor series as a generic property of holomorphic functions, Adv. Math. 157 (2001), no. 2, 138-176.

[25] A. Melas and V. Nestoridis, On various types of universal Taylor series, Complex Variables Theory Appl. 44 (2001), no. 3, 245-258.

[26] A. Melas, V. Nestoridis, and I. Papadoperakis, Growth of coefficients of universal Taylor series and comparison of two classes of functions, J. Anal. Math. 73 (1997), 187-202.

[27] J. Müller, V. Vlachou, and A. Yavrian, Universal overconvergence and Ostrowski-gaps, Bull. London Math. Soc. 38 (2006), no. 4, 597-606.

[28] V. Nestoridis, Universal Taylor series, Ann. Inst. Fourier (Grenoble) 46 (1996), no. 5, 1293-1306 (English, with English and French summaries).

[29] _ An extension of the notion of universal Taylor series, Computational methods and function theory 1997 (Nicosia), Ser. Approx. Decompos., vol. 11, World Sci. Publ., River Edge, NJ, 1999, pp. 421-430.

[30] V. Nestoridis, A strong notion of universal Taylor series, J. London Math. Soc. (2) 68 (2003), no. $3,712-724$.

[31] _ Non extendable holomorphic functions, Math. Proc. Cambridge Philos. Soc. 139 (2005), no. 2, 351-360.

[32] A. I. Seleznev, On universal power series, Mat. Sbornik N.S. 28(70) (1951), 453-460 (Russian).

[33] A. Zygmund, Trigonometric series. Vol. I, II, Cambridge Mathematical Library, Cambridge University Press, Cambridge, 1988. Reprint of the 1979 edition. 